\title{
Desarrollo de la escritura para la codificación y decodificación en niños de primaria de Mérida, Yucatán
}

\author{
Development of writing for encoding and decoding in primary school \\ children from Mérida, Yucatán

\section{Desenvolvimento da escrita para codificação e decodificação em crianças do ensino fundamental de Mérida, Yucatán} \\ Rutilio Nava Martínez \\ Universidad Modelo, México \\ rutilio.navam@modelo.edu.mx \\ https://orcid.org/0000-0001-9798-5560
}

\section{Resumen}

El objetivo del presente trabajo fue analizar el desarrollo y consolidación de la escritura en niños. Se evaluó a 546 niños entre la edad de 6 a 12 años utilizando pruebas de escritura y lectura con el fin de medir la cantidad de errores ortográficos. En los resultados se encontró que los niños de grados inferiores cometen, de forma frecuente, errores de ortografía natural (modificación que altera la fonología de la palabra, errores heterófonos); y que todos los niños cometen errores de ortografía arbitraria (sustitución de una letra homófona). Particularmente, se observó que los niños de segundo grado no identifican las palabras mal escritas y que son especialistas en el uso de grafías dominantes para representar su respectivo fonema. Los errores heterófonos y homófonos disminuyeron en los niños de cuarto grado, aquí hubo un mayor dominio de la relación fonema-grafema y viceversa; además, la etapa ortográfica se observó a esta edad, ya que los niños identificaron una mayor cantidad de palabras mal escritas, en comparación con los niños de segundo grado. Los niños de sexto grado mostraron una automatización para escribir e identificar unidades lexicales como ción, lo que significa que pueden leerlas como un todo y no como una secuencia de unidades 


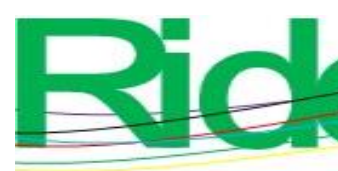

Revista Iberoamericana para la
Investigación y el Desarrollo Educativo
ISSN $2007-7467$

grafemas-fonemas. Por último, el aprendizaje de la escritura en el idioma español es rápido, la transición de la etapa alfabética parcial a la ortográfica toma dos años. En algunos casos, sin embargo, los individuos muestran dificultades o problemas (dislexia), por lo que siguen cometiendo errores ortográficos naturales, a pesar de estar expuestos a la misma cantidad de textos que sus pares. Estos individuos con problemas de aprendizaje fueron notorios en sexto grado.

Palabras clave: codificación y decodificación, consolidación de la escritura, desarrollo de la escritura, errores ortográficos, ortografía, pruebas de lectura y escritura.

\section{Abstract}

The objective of this work was to analyze the development and consolidation of writing in children. 546 children between the age of 6 to 12 years were evaluated using writing and reading tests in order to measure the number of spelling errors. In the results, it was found that children in lower grades frequently make natural spelling errors (modification that alters the phonology of the word, heterophone errors); and that all children make arbitrary spelling errors (substitution of a homophone letter). In particular, it was observed that second grade children do not identify misspelled words and that they are specialists in the use of dominant spellings to represent their respective phoneme. Heterophone and homophone errors decreased in fourth grade children, here there was a greater dominance of the phonemegrapheme relationship and vice versa; furthermore, the spelling stage was observed at this age, since the children identified a greater number of misspelled words, compared to the second-grade children. The sixth graders showed an automation for writing and identifying lexical units as -ción, which means that they can read them as a whole and not as a sequence of grapheme-phoneme units. Finally, learning to write in the Spanish language is fast, the transition from the partial alphabetic stage to the spelling one takes two years. In some cases, however, individuals show difficulties or problems (dyslexia), so they continue to make natural spelling errors, despite being exposed to the same amount of text as their peers. These individuals with learning disabilities were noticeable in sixth grade.

Keywords: writing development, writing consolidation, spelling, spelling errors, orthography, reading and writing tests. 


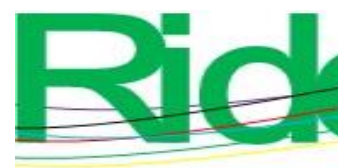

Revista Iberoamericana para la
Investigación y el Desarrollo Educativo
ISSN $2007-7467$

\section{Resumo}

O objetivo deste trabalho foi analisar o desenvolvimento e a consolidação da escrita em crianças. 546 crianças de 6 a 12 anos foram avaliadas por meio de testes de escrita e leitura para medir o número de erros ortográficos. Nos resultados, constatou-se que crianças de séries iniciais cometem erros ortográficos naturais com frequência (modificação que altera a fonologia da palavra, erros de heterofone); e que todas as crianças cometem erros ortográficos arbitrários (substituição de uma letra homófona). Em particular, observou-se que as crianças da segunda série não identificam palavras com erros ortográficos e são especialistas no uso da grafia dominante para representar seus respectivos fonemas. Os erros heterófonos e homófonos diminuíram nas crianças da $4^{\mathrm{a}}$ série, aqui houve maior dominância da relação fonema-grafema e vice-versa; além disso, o estágio de grafia foi observado nessa idade, uma vez que as crianças identificaram um número maior de palavras incorretas em relação aos da segunda série. Os alunos da sexta série apresentaram uma automação para a escrita e identificação das unidades lexicais como -ção, o que significa que podem lê-las como um todo e não como uma sequência de unidades grafema-fonema. Por fim, aprender a escrever na língua espanhola é rápido, a transição do estágio alfabético parcial para o de ortografia leva dois anos. Em alguns casos, porém, os indivíduos apresentam dificuldades ou problemas (dislexia), por isso continuam a cometer erros ortográficos naturais, apesar de serem expostos à mesma quantidade de texto que seus pares. Esses indivíduos com deficiência de aprendizagem eram perceptíveis na sexta série.

Palavras-chave: codificação e decodificação, consolidação da escrita, desenvolvimento da escrita, erros ortográficos, testes de ortografia, leitura e escrita.

Fecha Recepción: Octubre 2020

Fecha Aceptación: Marzo 2021 


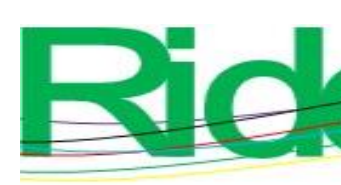

Revista Iberoamericana para la Investigación y el Desarrollo Educativo ISSN $2007-7467$

\section{Introducción}

La lectura y escritura implican un complejo sistema que relaciona a las palabras, continentes significados de distinto nivel de abstracción del entorno concreto del individuo, con una expresión gráfica organizada bajo determinada configuración. Es una habilidad que se adquiere en función del desarrollo intelectual del individuo y neurológicamente implica adecuaciones relacionadas con la adquisición de conceptos, la memorización y el manejo de patrones ortográficos de las palabras, el establecimiento de asociaciones de significados con signos organizados y la interpretación de la información.

Los modelos del aprendizaje de la lectoescritura explican el proceso sistemático de la adquisición y consolidación de la ortografía en los individuos. Al respecto, la autora Ehri (2005) realizó una revisión de todos los modelos que continúan utilizándose. Y enseguida propuso una serie de fases del desarrollo lector. La primera fase, conocida como la prealfabética, consiste en identificar las palabras o letras como señales visuales. En la segunda fase, alfabética parcial, los individuos conocen el nombre de las letras y las identifican; sin embargo, los sujetos aún carecen de habilidades de decodificación. En la tercera fase, alfabética completa, las personas desarrollan la estrategia de decodificación, formando conexiones entre grafemas y fonemas, y viceversa. Finalmente, la fase ortográfica consiste en el almacenamiento de secuencias de combinaciones de unidades grafo-fonémicas, incluyendo morfemas, rimas, palabras monosilábicas y polisilábicas.

Ahora bien, el proceso del aprendizaje de la lectura no es igual en todos los niños (Matute, González y Guajardo, 2012). Esto significa que no todos los individuos tienen las mismas facilidades o habilidades para el proceso.

Una correcta escritura depende de mecanismos de memoria visual. El almacenamiento de unidades léxicas facilita el reconocimiento de palabras y patrones ortográficos (Ferroni, Mena y Diuk, 2016). Por lo tanto, ya sea para escribir bien una palabra o para identificarla impresa, así como para un buen desarrollo de la lectoescritura, se apela a la misma huella viso-ortográfica almacenada en la memoria a largo plazo (Gómez et al., 2014).

Cabe destacar que si los mecanismos de automatización de un individuo no llegan a darse con la eficiencia esperada según su edad, esto traerá como consecuencia algunas complicaciones, por ejemplo, requerir de un mayor tiempo para la lectura de palabras que alguien sin dificultades (Afonso, Suárez y Cuetos, 2020; Suárez y Cuetos, 2013); también la 


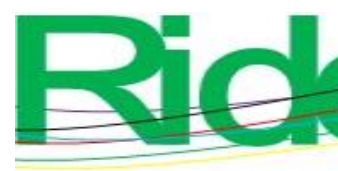

Revista Iberoamericana para la
Investigación y el Desarrollo Educativo
ISSN $2007-7467$

incapacidad de identificar errores ortográficos, que conduce a la aceptación de las palabras con errores ortográficos homófonos (E. Hm) (sustitución de una letra por otra con el mismo fonema) como palabras reales (González, Barrios, Gómez y Zarabozo, 2017; González, Gómez y Rodríguez, 2013; Guàrdia, Peró, Zarabozo, González y Gudayol, 2015).

Por lo que el objetivo de este trabajo es analizar el proceso de adquisición y consolidación de la lectoescritura en 546 individuos de $2 .^{\circ}(164), 4 .^{\circ}$ (184) y 6. ${ }^{\circ}$ (198) grado de primaria del municipio de Mérida, Yucatán, mediante pruebas de escritura y lectura. Se correlacionó el número de errores ortográficos escritos con las variables: el uso de grafías inconsistentes y la detección de errores ortográficos al leer.

\section{Metodología}

Participaron, de manera voluntaria, con el consentimiento informado por escrito por el director de la escuela y del profesor de cada grupo, 546 niños: 164 de $2 .^{\circ}$ grado, 184 de $4 .^{\circ}$ grado y 198 de $6 .^{\circ}$ grado. Todos los niños procedían de escuelas públicas de zona urbana y de diferentes niveles socioeconómicos: bajo, medio y alto.

Las escuelas fueron seleccionadas de manera aleatorizada. Tal y como se verificó, los planes de estudio de los centros educativos seleccionados siguen el plan nacional de educación dictado por la Secretaría de Educación Pública [SEP] (2017). Por último, las escuelas seleccionadas tienen una biblioteca, al igual que una clase de lectura de libros.

Los requisitos de participación fueron los siguientes: que los niños sepan leer y escribir; no recursar un año escolar; que la lengua materna fuera el español; ausencia de diagnóstico de algún trastorno o padecimiento de enfermedad neurológica y psiquiátrica, y el consentimiento del niño.

Consecuentemente, se excluyeron a 33 niños de 2. ${ }^{\circ}$ (no sabían leer y escribir), 10 niños de $4 .^{\circ}$ (uno era repetidor y nueve se negaron a participar) y cuatro niños de $6 .^{\circ}$ (uno padecía de problemas neurológicos y tres se negaron a participar). En la tabla 1 se muestran las características de los participantes. 

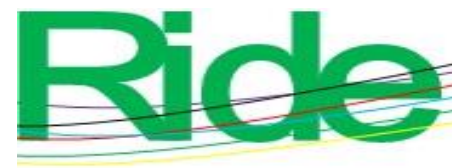

Revista Iberoamericana para la Investigación y el Desarrollo Educativo

ISSN $2007-7467$

En esta prueba se evaluaron los errores ortográficos detectados (ED) y las falsas detecciones (FD), esto es, las palabras escritas correctamente seleccionadas como mal escritas.

\section{Procedimiento}

Los niños fueron evaluados en sus aulas con la presencia del profesor del grado, y en algunas ocasiones con la presencia del director de la escuela, con una duración de entre 10 y 15 minutos. El autor del proyecto fue el que aplicó todas las evaluaciones.

El orden de presentación de las pruebas fue contrabalanceado. Cada tarea fue precedida de varios ejemplos con la intención de asegurar que el niño entendiera las instrucciones.

\section{Resultados}

\section{Dictado de palabras}

En la tabla 2 se observan los resultados descriptivos de la tarea de dictado de palabras. Se muestra el porcentaje de niños que cometieron errores ortográficos, tanto heterófonos como homófonos.

Se encontró que todos los niños cometieron al menos un E. Hm. Algo igualmente relevante fue que la mayoría de los niños de $2 .^{\circ}$ cometieron al menos un E. Ht (véase tabla 2). 
Tabla 2. Resultados descriptivos del dictado de palabras

\begin{tabular}{|l|l|l|l|l|l|l|}
\hline \multirow{2}{*}{} & \multicolumn{2}{|c|}{$2 .^{\circ}$ grado } & \multicolumn{2}{c|}{$4^{\circ}{ }^{\circ}$ grado } & \multicolumn{2}{c|}{ 6. $^{\circ}$ grado $^{\mathrm{b}}$} \\
\cline { 2 - 7 } & $\mathrm{E} . \mathrm{Hm}^{\mathrm{a}}$ & $\mathrm{E} . \mathrm{Ht}^{\mathrm{b}}$ & $\mathrm{E} . \mathrm{Hm}^{\mathrm{a}}$ & $\mathrm{E} . \mathrm{Ht}^{\mathrm{b}}$ & $\mathrm{E} . \mathrm{Hm}^{\mathrm{a}}$ & E. $\mathrm{Ht}^{\mathrm{b}}$ \\
\hline \% niños & $100 \%$ & $90.08 \%$ & $100 \%$ & $63.79 \%$ & $100 \%$ & $27.32 \%$ \\
\hline Media & 44.82 & 4.52 & 31.71 & 1.52 & 20.76 & 0.5 \\
(DE) & $(6.32)$ & $(3.9)$ & $(9.95)$ & $(2.07)$ & $(9.49)$ & $(1.21)$ \\
\hline Curtosis & 1.76 & 0.09 & -0.68 & 18.53 & 0.06 & 32.15 \\
\hline Skewness & -0.57 & 0.97 & -0.21 & 3.33 & 0.46 & 4.76 \\
\hline
\end{tabular}

E. $\mathrm{Hm}=$ Errores homófonos; E. HT = Errores heterófonos; \% niños = Porcentaje de niños que cometen E. Hm y E. Ht; DE = Desviación estándar. a = valor máximo 52. b = valor máximo 19. Fuente: Elaboración propia

Con la intención de averiguar cómo escriben los niños de primaria, se comparó la probabilidad de error de las grafías que pertenecen al mismo fonema. Para el análisis se utilizó la $U$ de Mann-Whitney, porque las variables no cumplieron la normalidad de acuerdo con la prueba Kolmogórov-Smirnov.

En los niños de 2. ${ }^{\circ}$ se encontró que, en el fonema /s/, la letra $s$ fue mejor escrita que la letra $c(U=3859, z=-7.841, p<0.001)$ y la letra $z(U=675, z=-13.706, p<0.001)$; asimismo, la letra $c$ obtuvo menos errores que la letra $z(U=5303, z=-6.942, p<0.001)$. En el fonema /b/, los niños fueron más asertivos en el uso de la letra $b$ en comparación con la letra $v(U=6634.5, z=-3.243, p=0.001)$. En el fonema $/ \mathrm{j} /$, los niños escribieron mejor la letra $j$ que la letra $g(U=6376, z=-3.740, p<0.001)$. Y en el fonema /y/, la letra $l l$ fue mejor escrita que la letra $y(U=2521, z=-10.487, p<0.001)$ (véase figura 1).

En los niños de cuarto grado de primaria se encontró que, en el fonema /s/, la letra $s$ obtuvo menos probabilidad de error que la letra $z(U=11300.5, z=-4.192, p<0.001)$; del mismo modo, la letra $c$ fue mejor escrita que la letra $z(U=11723, z=-3.758, p<0.001)$. En el fonema $/ y /$, los niños fueron más asertivos con la letra $l l$ que con la letra $y(U=6581, z=$ -9.646, $p<0.001)$. No hubo diferencias significativas en la letra $s$ en comparación con la letra $c(U=14965.5, z=-0.186, p=0.852)$; en la letra $b$ con la letra $v(U=13881, z=-1.376$, $p=0.169)$, y en la letra $j$ con la letra $g(U=14662, z=-0.565, p=0.572)$, ya que los niños tuvieron la misma probabilidad de error (ver figura 2). 


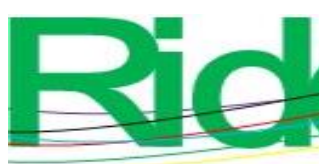

\section{Revista Iberoamericana para la Investigación y el Desarrollo Educativo ISSN 2007 - 7467}

$p<0.05)$. Por último, con la letra $l l$ se registró más asertividad que con la $y(U=10951.5, z$ $=-8.407, p<0.001$ ) para representar el fonema /y/ (véase la figura 3).

Con respecto a la letra silente $h$ y el acento, se observó que los niños de grados inferiores la usan menos, razón por la cual tienen una alta probabilidad de error; caso contrario de los niños de grados superiores.

\section{Dictado de no-palabras}

En la tabla 3 se muestra el porcentaje de número de niños que usaron una grafía para representar el fonema ante no-palabras; del mismo modo, se observa la probabilidad de uso de las grafías para representar el fonema.

Figura 3. Promedio de probabilidad de error de los niños de $6 .^{\circ}$ de primaria

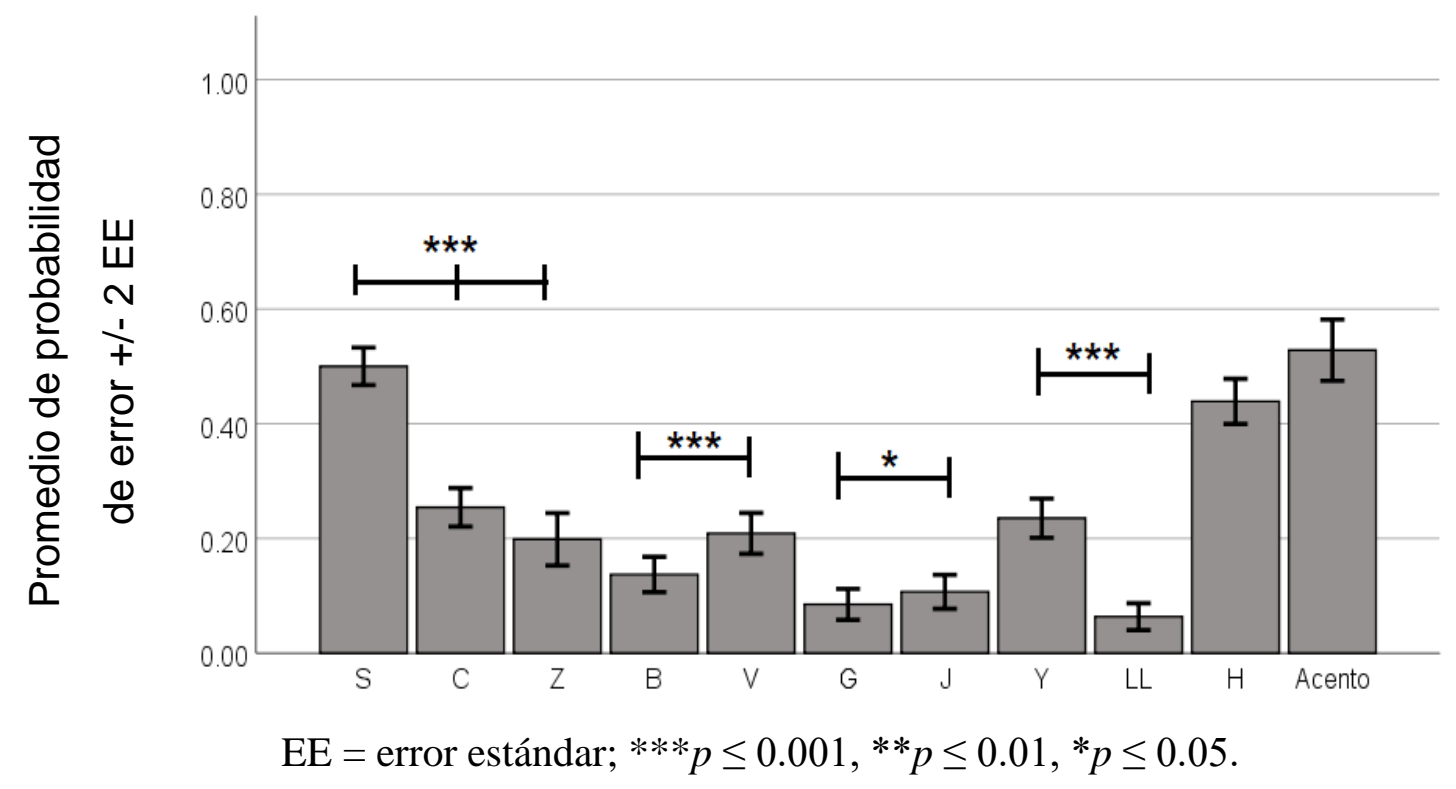

Fuente: Elaboración propia 


\section{Revista Iberoamericana para la Investigación y el Desarrollo Educativo ISSN 2007 - 7467}

Tabla 3. Probabilidad de uso de letras en el dictado de pseudopalabras

\begin{tabular}{|c|c|c|c|c|c|c|}
\hline & \multicolumn{2}{|c|}{ 2. ${ }^{\circ}$ grado } & \multicolumn{2}{|c|}{$4 .^{\circ}$ grado } & \multicolumn{2}{|c|}{$6 .^{\circ}$ grado } \\
\hline & $\%$ niños & Media (DE) ${ }^{a}$ & $\%$ niños & Media $(\mathrm{DE})^{\mathrm{a}}$ & $\%$ niños & Media (DE) ${ }^{a}$ \\
\hline$S$ & $99.23 \%$ & $0.846(0.22)$ & $99.42 \%$ & $0.729(0.21)$ & $100 \%$ & $0.604(0.17)$ \\
\hline $\mathrm{C}$ & $45.04 \%$ & $0.129(0.19)$ & $73.56 \%$ & $0.215(0.17)$ & $91.75 \%$ & $0.312(0.14)$ \\
\hline $\mathrm{Z}$ & $11.45 \%$ & $0.017(0.05)$ & $37.36 \%$ & $0.049(0.08)$ & $62.37 \%$ & $0.084(0.08)$ \\
\hline B & $91.6 \%$ & $0.729(0.33)$ & $94.83 \%$ & $0.644(0.32)$ & $98.97 \%$ & $0.685(0.23)$ \\
\hline V & $52.67 \%$ & $0.255(0.33)$ & $71.84 \%$ & $0.344(0.31)$ & $80.93 \%$ & $0.315(0.23)$ \\
\hline $\mathrm{G}$ & $51.91 \%$ & $0.336(0.39)$ & $74.71 \%$ & $0.432(0.36)$ & $85.05 \%$ & $0.481(0.34)$ \\
\hline $\mathrm{J}$ & $81.68 \%$ & $0.655(0.39)$ & $83.91 \%$ & $0.556(0.36)$ & $84.02 \%$ & $0.519(0.34)$ \\
\hline $\mathrm{Y}$ & $35.11 \%$ & $0.186(0.31)$ & $51.72 \%$ & $0.199(0.25)$ & $71.64 \%$ & $0.241(0.21)$ \\
\hline LL & $87.02 \%$ & $0.782(0.34)$ & $96.55 \%$ & $0.784(0.27)$ & $95.88 \%$ & $0.732(0.24)$ \\
\hline $\mathrm{H}^{\mathrm{b}}$ & $6.11 \%$ & & $55.17 \%$ & & $67.53 \%$ & \\
\hline Acento $^{b}$ & $11.45 \%$ & & $34.48 \%$ & & $60.31 \%$ & \\
\hline
\end{tabular}

$\%$ niños = Porcentaje de niños que usan la letra; $\mathrm{DE}=$ Desviación estándar. ${ }^{\text {a }}$ Probabilidad

de uso de la letra. ${ }^{\mathrm{b}}$ Letra que no tiene probabilidad de uso.

Fuente: Elaboración propia

Posteriormente, se buscó la relación entre el número de errores homófonos (NEHm) cometidos por los niños, de la prueba de dictado, y la probabilidad de uso de las grafías, de la prueba de dictado de pseudopalabras (véase la tabla 4). Para la relación se utilizó el estadístico de correlación de Spearman, ya que las variables no cumplieron con la prueba de normalidad de Kolmogórov-Smirnov. 


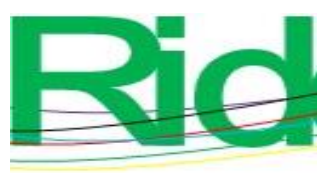

Revista Iberoamericana para la Investigación y el Desarrollo Educativo ISSN 2007 - 7467

Tabla 4. Coeficiente de correlación de Spearman: NEHm y el uso de grafías

\begin{tabular}{|l|l|l|l|}
\hline & NEHm..$^{\circ}$ grado & NEHm. $4 .^{\circ}$ grado & NEHm..$^{\circ}$ grado \\
\hline Letra S & $0.263^{* *}$ & $0.462^{* * *}$ & $0.430^{* * *}$ \\
\hline Letra C & $-0.288^{* * *}$ & $-0.398^{* * *}$ & $-0.210^{* *}$ \\
\hline Letra Z & -0.166 & $-0.483^{* * *}$ & $-0.467^{* * *}$ \\
\hline Letra B & -0.143 & -0.099 & 0.115 \\
\hline Letra $\mathrm{V}$ & 0.081 & 0.060 & -0.115 \\
\hline Letra G & $0.438^{* * *}$ & 0.021 & -0.085 \\
\hline Letra J & $-0.465^{* * *}$ & -0.069 & 0.085 \\
\hline Letra Y & 0.021 & $-0.166^{*}$ & -0.109 \\
\hline Letra LL & -0.131 & 0.138 & 0.082 \\
\hline Letra H & 0.031 & $-0.456^{* * *}$ & $-0.265^{* * *}$ \\
\hline Acento & $-0.267 * *$ & $-0.379^{* * *}$ & $-0.429 * * *$ \\
\hline
\end{tabular}

$\mathrm{NEHm}=$ Número de errores ortográficos homófonos. En la tabla se observan los coeficientes de correlación de Spearman. ${ }^{*} \mathrm{p}<0.05 ; * * \mathrm{p}<0.01 ; * * \mathrm{p}<0.001$. Fuente:

Elaboración propia

\section{Detección del error}

En la tarea de detección de errores ortográficos se observa que los niños de $2 .^{\circ}$ detectaron pocas palabras mal escritas y muchas FD; esto significa que algunos niños seleccionaron palabras que no tienen errores ortográficos como mal escritas. En los niños de 4. ${ }^{\circ}$ y $6 .^{\circ}$ de primaria se incrementaron los ED y se redujeron las FD (véase tabla 5). 
Tabla 5. Resultados de la prueba de detección del error

\begin{tabular}{|c|c|c|c|c|c|c|}
\hline & \multicolumn{2}{|c|}{$2 .^{\circ}$ grado } & \multicolumn{2}{|c|}{$4^{\circ}$ grado $^{\circ}$} & \multicolumn{2}{|c|}{$6 .^{\circ}$ grado } \\
\hline & $\begin{array}{l}\text { Media } \\
\text { (DE) }\end{array}$ & $\begin{array}{l}\text { Curt / } \\
\text { Skew }\end{array}$ & $\begin{array}{l}\text { Media } \\
(\mathrm{DE})\end{array}$ & $\begin{array}{l}\text { Curt I } \\
\text { Skew }\end{array}$ & $\begin{array}{l}\text { Media } \\
\text { (DE) }\end{array}$ & $\begin{array}{l}\text { Curt I } \\
\text { Skew }\end{array}$ \\
\hline $\begin{array}{l}\text { Errores } \\
\text { detectados }\end{array}$ & $\begin{array}{l}3.41 \\
(0.21)\end{array}$ & $\begin{array}{l}1.42 \quad / \\
1.05\end{array}$ & $\begin{array}{l}6.97 \\
(4.28)\end{array}$ & $\begin{array}{l}-0.49 \quad / \\
0.51\end{array}$ & $\begin{array}{l}11.54 \\
(3.96)\end{array}$ & $\begin{array}{l}-0.24 / \quad- \\
0.54\end{array}$ \\
\hline $\begin{array}{l}\text { Falsas } \\
\text { detecciones }\end{array}$ & $\begin{array}{l}3.78 \\
(0.39)\end{array}$ & $\begin{array}{l}9.15 \quad / \\
2.75\end{array}$ & $\begin{array}{l}1.36 \\
(2.23)\end{array}$ & $\begin{array}{l}21.62 \quad / \\
3.87\end{array}$ & $\begin{array}{l}0.61 \\
(0.91)\end{array}$ & $\begin{array}{l}5.43 \quad / \\
2.04\end{array}$ \\
\hline
\end{tabular}

$\mathrm{DE}=$ Desviación estándar; Curt $=$ Curtosis $;$ Skew $=$ Skewness .

Fuente: Elaboración propia

Para evaluar si la cantidad de comisión de errores ortográficos afecta la detección de los errores ortográficos, se correlacionó el NEHm cometido por los niños y las variables de la prueba de detección de errores: ED y FD. Se utilizó el estadístico de correlación de Spearman.

Se encontró que los niños de $2 .^{\circ}$ y de $4 .^{\circ}$ que cometieron más E. Hm tuvieron más FD $\left(2 .^{\circ} \rho=0.236, p<0.01 ; 4 .^{\circ} \rho=0.353, p<0.001\right)$; también se encontró que los niños de $4 .^{\circ} \mathrm{y}$ 6. ${ }^{\circ}$ que cometieron menos E. Hm identificaron más palabras mal escritas $\left(4 .^{\circ} \rho=-0.634, p<\right.$ $\left.0.001 ; 6 .^{\circ} \rho=-0.636, p<0.001\right)$.

No se encontró correlación entre el NEHm cometidos por los niños de $2 .^{\circ}$ y la detección de palabras mal escritas $(\rho=-0.157, p=0.075)$, tampoco entre el NEHm cometidos por los niños de $6 .^{\circ}$ y las FD $(\rho=0.061, p=0.396)$.

\section{Discusión}

En la presente investigación se analizó el proceso de adquisición y consolidación de la lectoescritura en niños de $2 .^{\circ}, 4 .^{\circ}$ y $6 .^{\circ}$ grado de primaria mediante pruebas de escritura y lectura.

De manera general, los resultados descriptivos muestran menor número de comisión de errores ortográficos, mayor uso de letras dominantes y mayor detección de palabras mal escritas en los niños de grados superiores en comparación con los de grados inferiores. Esta diferencia de grados es también encontrada en los trabajos de Defior, Martos y Herrera (2000), Defior, Jiménez y Serrano (2009) y Diuk, Borzone, Sánchez y Ferroni (2009). 


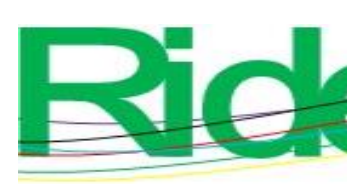

Revista Iberoamericana para la Investigación y el Desarrollo Educativo ISSN $2007-7467$

La prueba de dictado de palabras tiene la finalidad de identificar el desarrollo del conocimiento ortográfico y la edad en que empieza a tener importancia, además de identificar posibles problemas de aprendizaje de la lectoescritura. En esta prueba se obtienen dos resultados: la comisión de E. Ht y E. Hm.

No se detallará a profundidad sobre los E. Ht porque se utilizó un conjunto de fonemas (/t/,/m/, /r/, /l/, /a/, /e/, /i/, /o/ y /u/) que dan lugar a muy pocos errores ortográficos. Aunque sería interesante continuar con esta investigación y averiguar cuáles son las causas principales que conllevan al individuo a trasladar el fonema a una grafía no correspondiente al momento de escribir, como se realizó en el trabajo de Leal, Matute y Zarabozo (2005).

Una revisión superficial de los resultados de E. Ht muestran que más de $90 \%$ de los niños de 2. ${ }^{\circ}$ grado comete este tipo de error ortográfico. La investigadora Ehri (2005) menciona que, al ser nuevos en el ámbito de la lectura y escritura, los individuos carecen de habilidades para decodificar y codificar. Entonces, la comisión de E. Ht en la escritura se consideraría normal a este nivel escolar.

Caso contrario de los niños de $4 .^{\circ}$ y $6 .^{\circ}$ grado de primaria, quienes han tenido una mayor exposición a textos impresos, mínimo los libros de los grados anteriores que han cursado, por lo cual la comisión E. Ht podría considerarse un problema del aprendizaje de la lectoescritura (Baron et al., 2018; Morken y Helland, 2013; Suárez y Cuetos, 2015)

Este efecto es detallado en el trabajo de Landerl, Frith y Wimmer (1996), quienes señalan que el problema en individuos con dislexia se explica por un vínculo débil entre las representaciones fonológicas y ortográficas. Esto significa que ver una palabra escrita no evoca automáticamente el sonido interno de las palabras; tampoco el sonido de una palabra evoca automáticamente la representación ortográfica interna.

Con respecto a los E. Hm, se observa que todos los niños representaron el fonema, en la mayoría de las veces, por la misma letra, conocida como grafía dominante. Esto demuestra que los niños son especialistas en el uso de grafías dominantes en la escritura, tanto en palabras como no palabras. Las letras más utilizadas para representar el fonema /s/ es la $s$, el fonema /b/ es la $b$, el fonema /j/ es la $j$ y el fonema /y/ es la $l l$.

Estos resultados son similares a muchos trabajos de investigación (Diuk et al., 2009; Gómez et al., 2014; Jiménez et al., 2008). Por lo que se interpreta que los participantes cuentan con un buen dominio fonológico, pero tienen escaso conocimiento ortográfico (Sánchez, Diuk, Borzone y Ferroni, M., 2009; Signori y Borzone, 2003). 


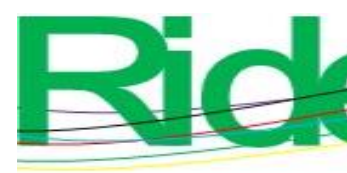

Revista Iberoamericana para la
Investigación y el Desarrollo Educativo
ISSN $2007-7467$

La especialización de las grafías en la escritura disminuye conforme aumenta el grado escolar (Defior et al., 2000; Gaintza, 2005), pero no desaparece, ya que los individuos de edad adulta siguen cometiendo este tipo de error ortográfico (Gómez et al., 2014; Moojen et al., 2020).

Un resultado relevante de la prueba de dictado de palabras es el aumento de probabilidad de error de la letra $s$ en niños de $6 .^{\circ}$ grado de primaria (véase la figura 3 ). Se considera que el aumento de error es por la invocación de pequeñas unidades léxicas, conocidos como morfemas. En la prueba de dictado de palabras se utilizaron palabras con terminación fonológica /sión/, normalmente las palabras que terminan con este sonido se representan en forma impresa con la grafía $c$, como -ción; pero en este trabajo las palabras se representaron con la grafía s, por ejemplo: precisión, versión e invasión.

En trabajos de lenguaje transparentes, en español (Suárez, Martínez y Cuetos, 2017) y en italiano (Angelelli, Marinelli, De Salvatore y Burani, 2017), se evaluaron el uso de morfemas en niños "normolectores" y "disléxicos". Los resultados muestran que, independientemente del grupo, los niños son más exactos en escribir las palabras y pseudopalabras que tienen como raíz morfemas frecuentes.

En el idioma español, -ción es un morfema frecuente y -sión es considerado un morfema infrecuente, ya que su uso depende de conocer algunas reglas de ortografía del idioma español, por lo que estos resultados dan indicios de que los niños de $6 .^{\circ}$ empiezan a escribir a través de lo almacenado en la memoria de largo plazo y no por lo fonológico, en otras palabras, con el conocimiento ortográfico o léxico.

Así pues, existe un almacenamiento de unidades léxicas pequeñas y grandes en la memoria a largo plazo que contribuyen a escribir correctamente (Ferroni et al., 2016; Gómez et al., 2014, Suárez et al., 2017) e identificar las palabras (Suárez y Cuetos, 2013; D'Alessio, Wilson y Jaincheco, 2019). Sin embargo, en este caso, y de acuerdo con nuestros resultados, interfiere en una correcta escritura.

A pesar de no ser uno de los objetivos, tampoco de las hipótesis planteadas, el resultado de la comisión de E. Ht de los niños de $6 .^{\circ}$ grado es relevante, ya que en este grado es notorio la diferencia entre los niños con problemas de aprendizaje de la lectoescritura, o dislexia, con sus pares. Los autores Suárez, Álvarez, Martínez, García y Cuetos (2015) mencionan que los individuos con problemas de aprendizaje de la lectoescritura muestran dificultad en adquirir y automatizar el código alfabético, al igual que en desarrollar 


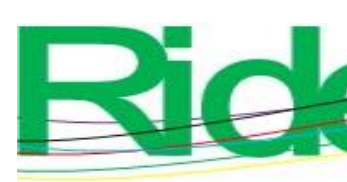

Revista Iberoamericana para la Investigación y el Desarrollo Educativo ISSN $2007-7467$

representaciones ortográficas de palabras. Sería interesante un estudio más exhaustivo del $30 \%$ de los niños que cometieron un E. Ht.

Los resultados de la prueba de identificación de errores ortográficos son similares a los trabajos de investigación de algunos autores (González et al., 2013; Guàrdia et al., 2015; González et al., 2017; Moojen et al., 2020), quienes indican que las personas con un buen léxico detectan más palabras con errores ortográficos y tienen diferentes respuestas electrofisiológicas (González et al., 2013) y activaciones neuroanatómicas (González et al., 2017; Guàrdia et al., 2015) que los individuos con un bajo nivel lexical.

Algo que refuerza el punto anterior es que en este estudio no existe una correlación entre el NEHm y ED en los niños de 2. ${ }^{\circ}$ grado: al tener poca experiencia lectora, los niños adivinan cuál es la palabra mal escrita (véase tabla 5). Esto significa que los niños no reconocen la parte ortográfica de las palabras.

El reconocimiento de palabras se aprecia en los niños de $4 .^{\circ}$ grado, con un aumento de detección de palabras mal escritas y una disminución de falsas detecciones, además de una disminución de grafías dominantes. Esto concuerda con los autores Jiménez et al. (2008), quienes mencionan que los niños españoles de 4. ${ }^{\circ}$ grado empiezan a escribir con el conocimiento ortográfico.

El proceso de aprendizaje de la escritura se asemeja al modelo de aprendizaje de la autora Ehri (2005). En la escritura se comienza en la fase alfabética parcial, donde los individuos empiezan a utilizar los sonidos de algunas letras para la formación de conexiones parciales, sílabas; sin embargo, carecen de habilidades de codificación, como se observa en este trabajo de investigación. Así, los niños recurren a diferentes estrategias como adivinar, utilizando claves fonéticas parciales o letras similares.

La etapa alfabética completa se visualiza en los niños de $4 .^{\circ}$ grado. En esta fase se desarrolla la habilidad de la decodificación y codificación, los sujetos recurren a la estrategia grafema-fonema y viceversa. Además, se visualiza la consolidación alfabética o etapa ortográfica, donde comienza el almacenamiento en la memoria a largo plazo, lo que facilita la automatización de las palabras para luego reconocerlas o leerlas a "golpe de vista": morfemas, rimas, palabras monosilábicas y ortografía de sílabas más frecuentes en palabras polisilábicas, por mencionar algunos ejemplos.

A medida que los lectores aprenden a leer palabras que comparten patrones de letras, los que simbolizan la misma combinación de fonemas de diferentes palabras, por ejemplo, 

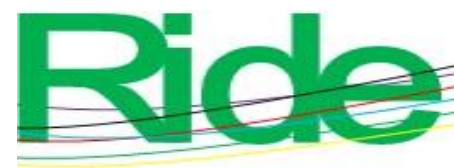

Revista Iberoamericana para la Investigación y el Desarrollo Educativo ISSN 2007 - 7467

canción, publicación, comunicación, se forma una unidad consolidada. El conocimiento de ción como una unidad consolidada significa que los lectores pueden leerlo como un todo y no como una secuencia de unidades grafemas-fonemas.

\section{Conclusiones}

Los errores ortográficos son comunes en el proceso de aprendizaje de la lectoescritura y van disminuyendo conforme hay una mayor exposición a los libros. Sin embargo, no desaparecen.

En sintonía con el proceso de adquisición y consolidación, similar a lo descrito por los modelos actuales, a pesar de la diferencia de idiomas, los niños de 2. empiezan el desarrollo de la conciencia fonológica, relación fonema-grafema y viceversa, pero tienen escaso conocimiento ortográfico, lo que les permite escribir la mayoría de las palabras conforme al sonido, características de la fase alfabética parcial.

Mientras que, en los niños de 4. ${ }^{\circ}$ grado se observa un mayor dominio de la conciencia fonológica y que empiezan a desarrollar el almacenamiento de unidades lexicales, tanto monosilábicas como polisilábicas. Aunque el almacenamiento de las combinaciones de letras no siempre es favorable. Por lo tanto, se considera que en este grado escolar es una transición entre la fase alfabética completa y la ortográfica.

Tomando en consideración lo anterior, podemos decir que el proceso de aprendizaje de la escritura en el idioma español es similar a las lenguas consideradas opacas, como el inglés, con la única diferencia de que las transiciones entre etapas son más rápidas. 


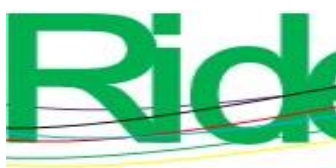

Revista Iberoamericana para la Investigación y el Desarrollo Educativo ISSN 2007-7467

\section{Referencias}

Afonso, O., Suárez, P. and Cuetos, F. (2020). Writing Impairments in Spanish Children with Developmental Dyslexia. Journal of Learning Disabilities, 53(2), 109-119.

Angelelli, P., Marinelli, C. V., De Salvatore, M. and Burani, C. (2017). Morpheme-based Reading and Spelling in Italian Children with Developmental Dyslexia and Dysorthography. Dyslexia, 23(4), 387-405.

Baron, L., Hogan, T., Alt, M., Gray, S., Cabbage, K., Green, S. and Cowan, N. (2018). Children with Dyslexia Benefit from Orthographic Facilitation During Spoken Word Learning. Journal of Speech, Language, and Hearing Research, 61(8), 2002-2014.

D'Alessio, M., Wilson, M. and Jaincheco, V. (2019). Morphological De-com-pos-it-ion Helps Recognize Low-er Frequency Words in Typically Developing Spanish Speaking Children. Journal of Psycholinguistic Research, 48(6), 1-22.

Dávalos, D., González, D., Kriscautzky, M. y Omaña, A. (2018). Lengua materna. Español. Primer grado. Ciudad de México, México: Secretaría de Educación Pública.

Defior, S., Jiménez, G. and Serrano, F. (2009). Complexity and lexicality effects on the acquisition of Spanish spelling. Learning and Instruction, 19(1), 55-65.

Defior, S., Martos, F. y Herrera, L. (2000). Influencia de las características del sistema ortográfico español en el aprendizaje de la escritura de palabras. Estudios de Psicología, 67, 55-64.

Diuk, B., Borzone, A., Sánchez, V. y Ferroni, M. (2009). La adquisición de conocimiento ortográfico en niños de $1^{\mathrm{er}}$ a $3^{\mathrm{er}}$ año de educación básica. Psykhe, 18(1), 61-71.

Ehri, L. (2005). Learning to read words: theory, findings, and issues. Scientific Studies of Reading, 9(2), 167-188.

Ferroni, M., Mena, M. y Diuk, B. (2016). Niveles de respuestas a una intervención en ortografía. Ciencias Psicológicas, 10(1), 55-61.

Gaintza, Z. (2005). Escritura de palabras de ortografía arbitraria en lengua castellana: evolución y métodos de instrucción. (Tesis doctoral). Euskal Herriko Unibertsitatea, País Vasco. Recuperado de https://dialnet.unirioja.es/servlet/tesis?codigo=130550

Gómez, F., González, A., Guàrdia, J., Peró, M., Zarabozo, D. y Zarabozo, D. (2014). Evaluación del conocimiento ortográfico en adultos jóvenes y su relación con la lectura. Revista Neuropsicología, Neuropsiquiatría y Neurociencias, 14(1), 40-67. 


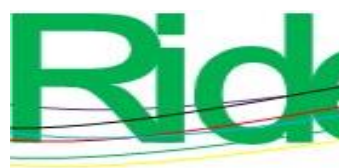

Revista Iberoamericana para la Investigación y el Desarrollo Educativo ISSN $2007-7467$

González, A., Barrios, F., Gómez, F. and Zarabozo, D. (2017). The supramarginal and angular gyri underlie orthographic competence in Spanish language. Brain and Language, 175, 1-10.

González, A., Gómez, F. y Rodríguez, E. (2013). Orthographic Recognition in Late Adolescents: An Assessment Through Event-Related Brain Potentials. Clinical EEG and Neuroscience, 45(2), 113-121.

Guàrdia, J., Peró, M., Zarabozo, D., González, A. and Gudayol, E. (2015). Effective connectivity of visual word recognition and homophone orthographic errors. Frontiers in Psychology, 6.

Jiménez, J., O'Shanahan, I., Tabraue, M., Artiles, C., Muñeton, M., Guzmán, R., Naranjo, F. y Rojas, E. (2008). Evolución de la escritura de palabras de ortografía arbitraria en lengua española. Psicothema, 20(4), 786-794.

Landerl, K., Frith, U. and Wimmer, H. (1996). Intrusion of orthographic knowledge on phoneme awareness: Strong in normal readers, weak in dyslexic readers. Applied Psycholinguistics, 17(1), 1-14.

Leal, F., Matute, E. y Zarabozo, D. (2005). La transparencia del sistema ortográfico del español de México y su efecto en el aprendizaje de la escritura. Estudios de Lingüísticas Aplicada, 23(42), 127-145.

Matute, E., González, A. L. y Guajardo, S. (2012). El sistema de escritura del español y sus efectos sobre las manifestaciones de la dislexia. En Matute, E. y Guajardo, S. (ed. ${ }^{\text {as}), ~}$ Dislexia: definición e intervención en hispanohablantes (1. a ed.) (pp. 37-46). México: El Manual Moderno.

Moojen, S., Gonçalves, H., Bassôa, A., Navas, A. L., De Jou, G. and Miguel, E. (2020). Adults with dyslexia: how can they achieve academic success despite impairments in basic reading and writing abilities? The role of text structure sensitivity as a compensatory skill. Annals of Dyslexia, 70(1), 115-140.

Sánchez, V., Diuk, B., Borzone, A. y Ferroni, M. (2009). El desarrollo de la escritura de palabras en español: Interacción entre el conocimiento fonológico y ortográfico. Interdisciplinaria, 26(1), 95-119.

Secretaría de Educación Pública [SEP]. (2017). Aprendizajes clave para la educación integral. Plan y programas de estudio para la educación básica . México: Secretaría de Educación Pública. 

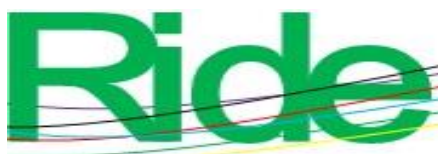

Signori, A. y Borzone, A. (2003). Aprendizaje de la lectura y escritura en español. El predominio de las estrategias fonológicas. Interdisciplinaria, 20(1), 5-30.

Suárez, P. and Cuetos, F. (2013). The Role of Morphology in Reading in Spanish-Speaking Children with Dyslexia. Spanish Journal of Psychology, 16, 1-7.

Suárez, P. and Cuetos, F. (2015). Reading difficulties in Spanish adults with dyslexia. Annals of Dyslexia, 65(1), 33-51.

Suárez, P., Alvárez, M., Martínez, C., García, N. and Cuetos, F. (2016). Reading prosody in Spanish dyslexics. Annals of Dyslexia, 66, 275-300.

Suárez, P., Martínez, C. and Cuetos, F. (2017). Morpheme-Based Reading and Writing in Spanish Children with Dyslexia. Frontiers in Psychology, 8, 1-9. 